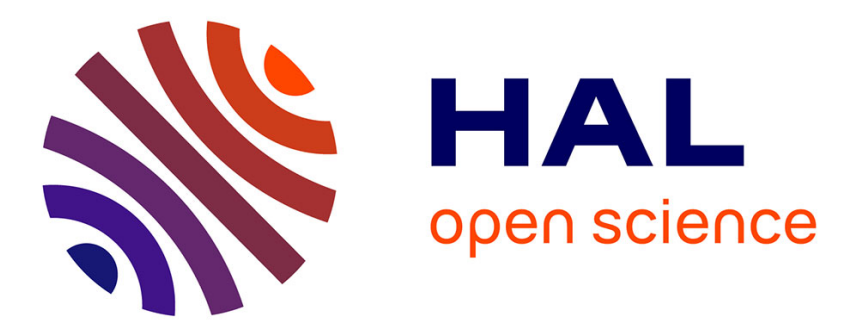

\title{
Subtle adjustments of the glucose-6-phosphate dehydrogenase (G6PD) mutation database and reference sequence.
}

Stéphane Mazières, Florence Petit, Jean-Michel Dugoujon, Xavier Iriart, Antoine Berry, Bernard Carme, Mathieu Nacher, Pascal Bailly, Jacques

Chiaroni

\section{To cite this version:}

Stéphane Mazières, Florence Petit, Jean-Michel Dugoujon, Xavier Iriart, Antoine Berry, et al.. Subtle adjustments of the glucose-6-phosphate dehydrogenase (G6PD) mutation database and reference sequence.. Blood Cells, Molecules and Diseases, 2014, 52 (1), pp.55-6. 10.1016/j.bcmd.2013.07.001. hal-00853976

\section{HAL Id: hal-00853976 https://hal.science/hal-00853976}

Submitted on 26 Aug 2013

HAL is a multi-disciplinary open access archive for the deposit and dissemination of scientific research documents, whether they are published or not. The documents may come from teaching and research institutions in France or abroad, or from public or private research centers.
L'archive ouverte pluridisciplinaire HAL, est destinée au dépôt et à la diffusion de documents scientifiques de niveau recherche, publiés ou non, émanant des établissements d'enseignement et de recherche français ou étrangers, des laboratoires publics ou privés. 


\section{Letter to the Editor}

\section{Subtle adjustments of the Glucose-6-phosphate dehydrogenase (G6PD) mutations database and reference sequence.}

\section{To the Editor:}

Reference sequences and mutation databases are essential for the development of molecularbased methods in human genetics. Lately, Minucci et al. [1] revised the glucose-6-phosphate dehydrogensase (G6PD) reference material from 131 bibliographic references, three previous databases, and the genomic reference sequence (GenBank accession number X55448.1). Deficiency in G6PD is the most common enzymatic insufficiency in human populations and clinical manifestations range from mild to severe: neonatal jaundice, acute hemolysis and chronic non-spherocytic hemolytic anemia prompted by infections or sudden onset of oxidative agents from for instance the ingestion of fava beans (Vicia faba) or anti-malarial treatments [2]. Given the clinical consequences, numerous studies and international projects are being carried out about the mechanisms leading to the numerous G6PD enzymatic variants, and in this context, the updated G6PD database provided in [1] is an precious substratum for lab protocols. Nonetheless, while setting our strategy from this material, we encountered two main hurdles that we would like to address.

In order to accurately design our sequencing primers, we started by manually relocating each of the 187 listed substitutions onto X55448.1 by the addition of 3350 to their respective genomic DNA nucleotide position (gDNA np) [1]. We then validated each targeted substitution according to the recognition enzyme site it should create or abolish. Occasionally, we have observed a mismatch between some nucleotides listed in [1] and the G6PD reference sequence X55448.1. For instance, the Rignano mutation involving a G or A was expected at np13337 in X55448.1. However, np13337 is a $\mathrm{C}$ while the preceding nucleotide is a G. Relocation of the substitutions revealed that the G6PD mutation database mismatched with X55448.1 by one base at exons 3, 4 and 5 . We presume that this numbering shift originated from the update of the former X55448 involving a deletion of one T before exon 3 at np8850 [1]. In order to asses our suspicion, we then ran a multiple sequence alignment of several G6PD reference sequences using BioEdit version 7.0.5.3 [3]: ultimate (X55448.1), former 
(X55448), Homo sapiens RefSeqGene (NG_009015.1) and coding DNA (X03674.1). Sequence

alignment confirmed that the entire block encompassed between np8850 and np14575 of X55448.1 is shifted one-base toward the left. This "sliding-effect" is then counter-balanced from intron 5 by the addition of a C at np14575; another major update of the former X55448 [1]. Supplementary Table 1 lists the 37 substitutions affected by the incident among which 22 are unambiguous.

Concomitantly, the multiple sequence alignment showed that X55448.1 carried an A at np13503 and a G at np14226 (Table 1), characteristic of the $\mathrm{A}^{-(202)}$ variant [4]. This is problematic since all G6PD enzymatic variants are given according to the wild-type B variant, and the reference sequence should mirror it (Figure 1). We sequenced exons 4 and 5 in two male samples free from G6PD deficiency using the primers previously published [5]. Capillary electrophoresis was carried out on an automated fluorescence-based ABI PRISM ® 3130 XL genetic analyzer (Applied Biosystem, Forster City, CA, USA) according to the manufacturer's protocol. Sequencing and alignment of our two samples confirm the non-ancestral allelic state of the G6PD reference sequence X55448.1 of the NCBI BioSystems database [6].

Additionally, we found four minor misprints: the Nashville-Anaheim-Portici and Georgia variants were spotted at gDNA np13447 and 13560 while they are actually at gDNA np13477 and 13583. GDNA coordinate of the $\mathrm{A}^{-(680)}$ mutation was erroneously written 120228 , and the Acrokorinthos variant has been labeled in exon 12 while it is in exon 5 [7] (Supplementary Table1).

In conclusion, we authenticated that the nucleotide substitutions of exons 3,4 , and 5 from the latest mutation database have been erroneously numbered by +1 , and the updated $G 6 P D$ reference sequence appears to be non-wild-type. Four minor misprints have also been spotted. Herein we provide the slight modifications to favor the standardization of the G6PD reference material: firstly subtracting 1 to the gDNA coordinates of the nucleotide variations of exons 3, 4, and 5; and secondly, reversing two punctual polymorphisms to turn over the reference sequence to the wild-type allele status. G6PD reference sequence and mutation database of [1] remain essential to the identification of enzymatic variants in areas of malaria exposure from molecular-based attempts. 
Acknowledgement:

This work has benefited from an Investissement d'Avenir grant of the ANR (CEBA: ANR-10-LABX0025). 


\section{References}

[1] A. Minucci, K. Moradkhani, M.J. Hwang, C. Zuppi, B. Giardina, and E. Capoluongo, Glucose-6phosphate dehydrogenase (G6PD) mutations database: review of the "old" and update of the new mutations. Blood Cells Mol Dis 48 (2012) 154-65.

[2] E. Beutler, Glucose-6-phosphate dehydrogenase deficiency: a historical perspective. Blood 111 (2008) 16-24.

[3] T.A. Hall, BioEdit: a user-friendly biological sequence alignment editor and analysis program for Windows 95/98/NT. Nucl. Acids. Symp. Ser. 41 (1999) 95-98.

[4] T.J. Vulliamy, M. D'Urso, G. Battistuzzi, M. Estrada, N.S. Foulkes, G. Martini, V. Calabro, V. Poggi, R. Giordano, M. Town, and et al., Diverse point mutations in the human glucose-6-phosphate dehydrogenase gene cause enzyme deficiency and mild or severe hemolytic anemia. Proc Natl Acad Sci U S A 85 (1988) 5171-5.

[5] A. Minucci, L. Gentile, C. Zuppi, B. Giardina, and E. Capoluongo, Rapid and simple identification of the commonest glucose-6-phosphate dehydrogenase (G6PD) Italian mutations: from DNA extraction to genotyping. Clin Chim Acta 413 (2012) 1018-9.

[6] L.Y. Geer, A. Marchler-Bauer, R.C. Geer, L. Han, J. He, S. He, C. Liu, W. Shi, and S.H. Bryant, The NCBI BioSystems database. Nucleic Acids Res 38 (2010) D492-6.

[7] A. Drousiotou, E.H. Touma, N. Andreou, J. Loiselet, M. Angastiniotis, B.C. Verrelli, and S.A. Tishkoff, Molecular characterization of G6PD deficiency in Cyprus. Blood Cells Mol Dis 33 (2004) 25-30. 
Stéphane Mazières

Florence Petit

Aix Marseille Université, CNRS, EFS-AM, ADES UMR7268, 13344, Marseille, France.

Jean-Michel Dugoujon

Université Paul Sabatier, CNRS, AMIS UMR5288, 31073, Toulouse, France

Xavier Iriart

Antoine Berry

Service de Parasitologie-Mycologie, Centre Hospitalier Universitaire de Toulouse / INSERM UMR1043/CNRS UMR5282/Université de Toulouse UPS, Centre de Physiopathiologie de Toulouse Purpan (CPTP), 31300, Toulouse, France

Bernard Carme, ${ }^{5}$

Mathieu Nacher, ${ }^{5}$

CIC-EC Antilles Guyane CIE 802 Inserm, Centre Hospitalier Andrée Rosemon

Equipe EPaT EA 3593, Université des Antilles et de la Guyane, Cayenne, Guyane française.

Pascal Bailly Jacques Chiaroni $^{*}$

${ }^{3}$ Établissement Français du Sang Alpes Méditerranée, 13005, Marseille, France Email address: jacques.chiaroni@efs.sante.fr

*Corresponding author. Fax: +33(0)4 91189350 
Table 1 Nucleotide variations in a set of G6PD reference sequences and samples. Coding (cDNA) and genomic (gDNA) coordinates correspond to the locations on X03674.1 and X55448.1 respectively.

\begin{tabular}{lcccc}
\hline Sequence & GenBank & 202G->A & 376A->G & Allele \\
& Accession number & (cDNA np672, gDNA np13503) & (cDNA np846, gDNA np14226) \\
\hline H. sapiens G6PD RefSeqGene & NG_009015.1 & G & A & B \\
cDNA & X03674.1 & $\cdot$ & $\cdot$ & B \\
Our samples & N/A & $\cdot$ & B & B \\
gDNA H. sapiens G6PD gene & X55448.1 & A & A \\
\hline
\end{tabular}


Supplementary Table 1 List of the 37 G6PD mutations whose gDNA numbering should be modified by minus 1 as inferred from X55448.1. The four misprints (underlined) were detected after comparison with X03674.1 and recognition enzyme sites [1].

\begin{tabular}{|c|c|c|c|c|c|c|}
\hline Substitution & Exon & Mutation name & gDNA np & Observed nucleotide & Accurate nucleotide & $\begin{array}{l}\text { Rectified } \\
\text { gDNA np }\end{array}$ \\
\hline \multicolumn{7}{|c|}{ 1.One-base shifting } \\
\hline $130 \mathrm{G}>\mathrm{A}$ & 3 & Rignano & 9987 & $13337 \mathrm{C}$ & $13336 \mathrm{G}$ & 9986 \\
\hline $131 \mathrm{C}->\mathrm{G}$ & 3 & Orissa & 9988 & $13338 \mathrm{C}$ & $13337 \mathrm{C}$ & 9987 \\
\hline $143 \mathrm{~T}->\mathrm{C}$ & 3 & Aures & 10000 & $13350 \mathrm{C}$ & $13349 \mathrm{~T}$ & 9999 \\
\hline $148 \mathrm{C}->\mathrm{T}$ & 3 & Kambos & 10005 & $13355 \mathrm{C}$ & $13354 \mathrm{C}$ & 10004 \\
\hline $159 \mathrm{G}->\mathrm{C}$ & 4 & Kozukata & 10111 & $13461 \mathrm{~T}$ & $13460 \mathrm{G}$ & 10110 \\
\hline $169 \mathrm{C}->\mathrm{T}$ & 4 & Kamogawa & 10121 & $13471 \mathrm{G}$ & $13470 \mathrm{C}$ & 10120 \\
\hline $170 \mathrm{G}>\mathrm{A}$ & 4 & Palestrina & 10122 & $13472 \mathrm{G}$ & $13471 \mathrm{G}$ & 10121 \\
\hline $172 \mathrm{G}->\mathrm{A}$ & 4 & Metaponto & 10124 & $13474 \mathrm{~A}$ & $13473 \mathrm{G}$ & 10123 \\
\hline $179 \mathrm{~T}>\mathrm{C}$ & 4 & Costanzo & 10131 & $13481 \mathrm{~T}$ & $13480 \mathrm{~T}$ & 10130 \\
\hline 180_182del TCT & 4 & Amsterdam & $10132-34$ & 13482-84CTG & 13481-83ТCT & $10131-33$ \\
\hline $185 \mathrm{C}->\mathrm{A}$ & 4 & Amazonia-Musashino & 10137 & $13487 \mathrm{C}$ & $13486 \mathrm{C}$ & 10136 \\
\hline $196 \mathrm{~T}->\mathrm{A}$ & 4 & Songklanagarind & 10148 & $13498 \mathrm{~T}$ & $13497 \mathrm{~T}$ & 10147 \\
\hline $202 \mathrm{G}->\mathrm{A}$ & 4 & Asahi, Hechi, $A^{-(202)}$, no name & 10154 & $13504 \mathrm{~T}$ & $13503 \mathrm{G}$ & 10153 \\
\hline
\end{tabular}


Supplementary table 1. Continued

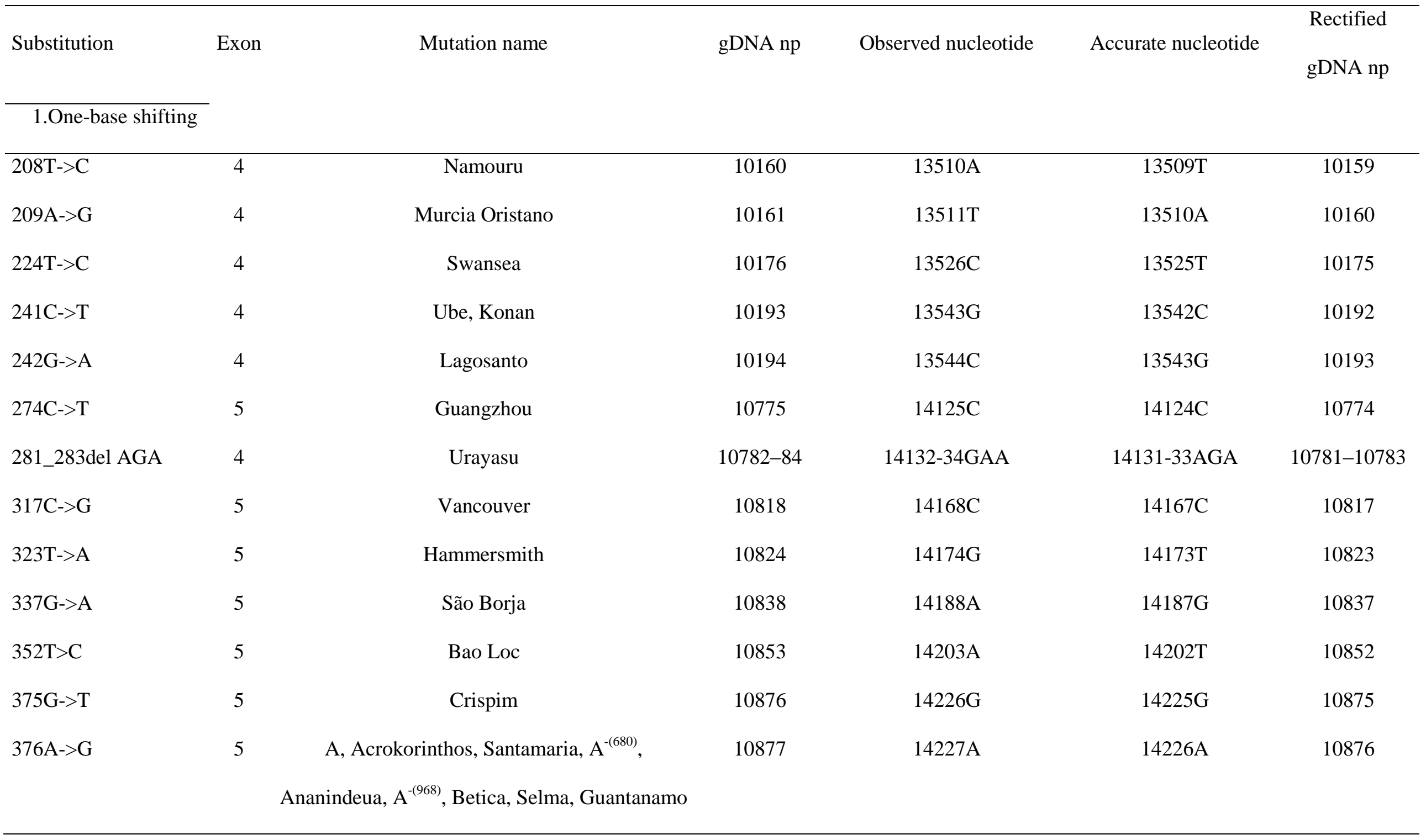


Supplementary table 1. Continued

\begin{tabular}{|c|c|c|c|c|c|c|}
\hline Substitution & Exon & Mutation name & gDNA np & Observed nucleotide & Accurate nucleotide & $\begin{array}{l}\text { Rectified } \\
\text { gDNA np }\end{array}$ \\
\hline \multicolumn{7}{|c|}{ 1.One-base shifting } \\
\hline 379G->T & 5 & Crispim & 10880 & $14230 \mathrm{C}$ & $14229 \mathrm{G}$ & 10879 \\
\hline $383 \mathrm{~T}->\mathrm{C}$ & 5 & Vanua Lava-Crispim-Salerno Pyrgos & 10884 & $14234 \mathrm{C}$ & $14233 \mathrm{~T}$ & 10883 \\
\hline $384 C>T$ & 5 & Crispim & 10885 & $14235 \mathrm{C}$ & $14234 \mathrm{C}$ & 10884 \\
\hline $392 \mathrm{G}->\mathrm{T}$ & 5 & Quing Yan & 10893 & $14243 G$ & $14242 \mathrm{G}$ & 10892 \\
\hline $404 \mathrm{~A}->\mathrm{C}$ & 5 & Cairo & 10905 & $14255 \mathrm{C}$ & $14254 \mathrm{~A}$ & 10904 \\
\hline $406 \mathrm{C}->\mathrm{T}$ & 5 & Valladolid & 10907 & $14257 \mathrm{G}$ & $14256 \mathrm{C}$ & 10906 \\
\hline $409 \mathrm{C}->\mathrm{T}$ & 5 & Belem & 10910 & $14260 \mathrm{~T}$ & $14259 \mathrm{C}$ & 10909 \\
\hline $442 \mathrm{G}->\mathrm{A}$ & 5 & Liuzhou & 10943 & $14293 \mathrm{~A}$ & $14292 \mathrm{G}$ & 10942 \\
\hline $466 \mathrm{G}->\mathrm{A}$ & 5 & Ilesha & 10967 & 14317A & $14316 \mathrm{G}$ & 10966 \\
\hline $473 \mathrm{G}>\mathrm{A}$ & 5 & Shenzen & 10974 & $14324 \mathrm{C}$ & $14323 \mathrm{G}$ & 10973 \\
\hline $477 \mathrm{G}>\mathrm{C}$ & 5 & Gond & 10978 & $14328 \mathrm{~A}$ & $14327 \mathrm{G}$ & 10977 \\
\hline \multicolumn{7}{|l|}{ 2.Misprints } \\
\hline 1178G->A & & Nashville, Anaheim, Portici & 13447 & $1648 \mathrm{G}$ & $16827 \mathrm{G}$ & 13477 \\
\hline
\end{tabular}


Supplementary table 1. Continued

\begin{tabular}{|c|c|c|c|c|c|c|}
\hline Substitution & Exon & Mutation name & gDNA np & Observed nucleotide & Accurate nucleotide & $\begin{array}{l}\text { Rectified } \\
\text { gDNA np }\end{array}$ \\
\hline \multicolumn{7}{|l|}{ 2.Misprints } \\
\hline \multirow[t]{3}{*}{$1284 C->A$} & & Georgia & 13560 & $1754 \mathrm{~A}$ & $16933 \mathrm{C}$ & 13583 \\
\hline & & $\mathrm{A}^{-(680)}$ & 120228 & & & $\underline{12028}$ \\
\hline & $\underline{5}$ & Acrokorinthos & & & & \\
\hline
\end{tabular}


Figure 1. Extract of X55448.1 with the main modifications turning over the sequence from the $\mathrm{A}^{-(202)}$ to the B variant at np13503 and np14226 (open boxes)

\begin{tabular}{|c|c|c|c|c|c|c|}
\hline & & gtc & $\mathrm{CC}$ & ggt & $=c t$ & \\
\hline & & & $\mathrm{CCC}$ & $\operatorname{atcc}$ & gctc & \\
\hline & $g c$ & $\operatorname{cctgctc}$ & gtacttct & tgagaccecc & attaccagcc & cccgtg \\
\hline & cag & gtccetcctg & ctgtgctctg & ctgcgttttc & tccgccaatc & ata \\
\hline & & gagag & getttctcca & gtgtatttct & $\mathrm{caa}$ & \\
\hline & atct & ctgtcctaag & gcacaggggt & cccagcctgg & $\operatorname{ggc}$ & gto \\
\hline & ctc & tccetctg & gatgtgcaga & gctgctaaga & sctgaa & $\mathrm{CC}$ \\
\hline & ac & gacttctg & agggcaccct & ccctggacct & ccagggaaga & $\operatorname{ccctcc}$ \\
\hline & ra & ac & ggactcaaag & agaggggctg & catctgtct & gtgtgt \\
\hline & tgt & tcccaggec & accccagagg & agaagctcaa & gctggaggac & tectet \\
\hline & ta & $\operatorname{tgg} c$ & cagtacgatg & atgcagcctc & ctaccagcgc & tcaa \\
\hline & & & & & & \\
\hline
\end{tabular}

